\title{
Trabajo Social como Transdisciplina: hacia una teoría de la intervención
}

\author{
SOCIAL WORK AS TRANSDISCIPLINE: TOWARDS A THEORY OF INTERVENTION
}

Dra. María Belén Ortega (belenmim@gmail.com) Facultad de Comunicación, Historia y Ciencias Sociales, Universidad Católica de la Santísima Concepción (Concepción, Chile)

\begin{abstract}
Social work is assumed as transdisciplinary and because of that the aim of this paper is to reflect about the complex foundations of intervention theory, constructed from an organic and consistent profession. The idea is to contribute to the creation of a corpus that overcomes the technological tradition responding to three challenges: the creation of inclusive and collaborative processes with subjects, the transformation of diagnosis and intervention techniques in research resources, and the inclusion of symbols as an inseparable dimension from everyday practices and social structures of the subjects of study and intervention.
\end{abstract}

Key words: social work, transdiscipline, intervention theory, complexity, performativity.

\section{Resumen}

Desde el trabajo social asumido como transdisciplina se pretende reflexionar sobre las bases complejas necesarias para abrir caminos hacia una teoría de la intervención, construida desde una investigación coherente y orgánica a la profesión. La idea es aportar a la conformación de un corpus que supere y asuma la tradición tecnológica dando respuesta a tres desafíos: la conformación de procesos investigativos inclusivos y colaborativos con los sujetos, la reversibilidad de técnicas de diagnóstico e intervención en recursos de investigación, e incluir lo simbólico como dimensión fundamental e inseparable de las prácticas cotidianas y las estructuras sociales de los sujetos de estudio e intervención del trabajo social.

Palabras clave: trabajo social, transdisciplina, teoría de la intervención, complejidad, performatividad.

\section{Introducción}

Desde la academia, vemos cómo el trabajo social busca la manera de integrar la investigación en su cuerpo disciplinario, en un esfuerzo por alcanzar al resto de ciencias sociales y humanistas que, en toda la región latinoamericana, viene realizando un trabajo reflexivo para lograr investigaciones cualitativas, complejas y atingentes a las necesidades de comprensión, comunicación y participación que tienen los sujetos de estudio e intervención social: "El trabajo social no puede quedar perplejo -confundido- ante los vertiginosos cambios e innovaciones que las sociedades contemporáneas arrastran" (Vélez 2003:42).

De este modo, la disciplina debe vivir dos procesos paralelos: por un lado, incorporar la búsqueda de conocimiento desde parámetros éticos y transformadores; $y$, por otro, encontrarse 
epistemológicamente con los nuevos paradigmas vigentes que demandan "una complejidad basada en un cambio de miradas sobre los sujetos para transformarlas en miradas con los sujetos; en una revisión deconstructiva, abierta y reflexiva de nuestros conceptos y definiciones de lo qué es y puede ser posible; en el desmantelamiento de las fronteras disciplinares; y en la asunción de la subjetividad y la incertidumbre como variables permanentes e inevitables" (Ortega 2013:62), es decir, "en la cualitividad en sustitución del rigor encorsetador" (Sousa Santos 2003:33).

No obstante, y a pesar de que estas nuevas miradas de los sujetos/objetos no nos son tan ajenas, dado que de alguna manera la práctica profesional y las fuentes de apoyo teóricas y científicas nos han ido conformando como "un corpus complejo y transdisciplinario" (Quiroz 2010:168); hasta que no asumamos completamente el paso de profesión a disciplina, no lograremos ir al encuentro de una comprensión social que contemple "transformaciones contextuales, teoría social, enfoques epistemológicos, perspectivas éticas y valóricas" (Matus 2002:16) desde una conformación compleja como parte de nuestro ser y quehacer.

Por otra parte, no parece estar resultando sencillo pasar por ese proceso de disciplinarse, o construirse disciplina, con una historia fuertemente profesionalizada desde la permeabilidad y la heterodoxia que nos ha caracterizado, produciendo un desasosiego ontológico en el que nos preguntamos cómo vamos a ser productores de conocimiento y debate teórico.

De ahí, que la reflexión de los desafíos que proponemos a continuación parta de la siguiente premisa: ¿por qué no pensarnos como transdiciplina? Desde la concepción de Nicolescu, la transdisciplinariedad implica pensar en la construcción de saberes desde una perspectiva que sitúe "más allá y a través de las disciplinas" (Nicolescu 1996:37). El concepto no solo asume los principios de complejidad aflorados en el debate contemporáneo, sino que además abogan por una comprensión multidimensional de la realidad que determinan, o deben determinar, una metodología transdisciplinaria. Con la familiaridad con la que nos relacionamos con este planteamiento es que podemos asumir al trabajo social como transdisciplina, construir desde ahí y averiguar qué nos depara en el ámbito teórico y práctico.

\section{Los desafíos para una investigación desde el trabajo social}

La vigilancia epistemológica contempla el repensamiento de qué somos, qué hacemos, cómo lo hacemos, por qué lo hacemos y cómo nos vemos en prospectiva con las ciencias sociales y la sociedad misma. De este modo, nuestra principal tarea pasa tanto por la "reconceptualización del objeto en la dinámica de cambios y condiciones sociales, y resituar la crisis de la praxis en el mundo de la crisis misma de la ciencia, como dinámica en torno a los paradigmas de las ciencias sociales" (Cifuentes 2009:129), como por la redefinición de nuestra relación con el objeto y los sujetos, y ello contempla: "Sistemas teóricos, sistemas conceptuales, enfoques epistemológicos; y el eje conceptual metodología y método en que se abordan las categorías referentes e implicaciones metodológicas" (Camelo y Cifuentes 2006:181), tanto para la intervención como para la investigación.

En consideración a lo dispuesto, se aborda una serie de retos que pretende aportar a un andamiaje metodológico en investigación desde nuestra área, procurando la permeabilidad a otras disciplinas y pretendiendo ser orgánica a su sentido de ser: la intervención o la acción reflexiva. En este artículo, indagaremos una manera de encontrarnos con lo teórico desde el objeto y los sujetos del trabajo social que se conforme más allá de la reflexión de la praxis profesional, esto quiere decir, que el hacer teoría social desde la posición privilegiada de la intervención social no debe ser confundida con la 
sistematización de las prácticas; la diferenciación radica tanto en la metodología, como en los alcances del conocimiento, el cual trasciende al propio trabajo social. Para ello, relevamos "mejorar nuestras condiciones de reproducción transgrediendo las actualmente instaladas, proponiendo nuevos marcos y nuevos actores y abriéndonos a nuevos tipos de relaciones vinculantes" (Aquín 2006:18), a través de una superación que nos abra caminos hacia un corpus de conocimientos construido desde otra mirada, la que aporta la relación objeto-sujeto-acción.

El horizonte procurado no es alimentar una teoría "para" la intervención, sino una "teoría de la intervención" (Escobar 1999), requerida, también, por otras disciplinas afines como la antropología aplicada al desarrollo. Una teoría de la intervención nos ofrece fundamentos teóricos sobre lo social y de cómo se puede incidir en ello con objetivos de cambio en varios niveles, de lo micro a lo macro, incorporando necesidades, desigualdades y acciones sociales desde la cotidianidad de sus protagonistas, desde la vivencia de los acontecimientos, los problemas, los conflictos, las resistencias, las alternativas y las innovaciones, quebrando las fuerzas dispuestas como dispositivos bajo lógicas foucaultianas: "En cuanto entidad heterónoma de regulación biopolítica. Y cuando referimos a esa distinción heterónoma, estamos refiriendo a que la naturaleza de los componentes del dispositivo son diversos (legislación, arquitecturas, sujetos, agentes, entre otros), todos los cuales paradojalmente, configuran una unidad homogénea de normativización que regula diversos aspectos de la vida de la población" (Saavedra 2015:145).

En este sentido, queremos ver al trabajo social como una práctica provista de fortalezas ganadas por un carácter pragmático y un gran espectro de áreas de acción, que la llevan hacia la articulación teóricopráctica e investigativa-activa desde una posición distinta -podríamos decir privilegiada o complementaria- al resto de disciplinas sociales, ya que "posee un cierto tipo de conocimiento acerca de la vida cotidiana de los sujetos sobre los que interviene (...) ha tenido una tradición muy importante en la práctica de visualizar lo microsocial" (Carballeda 2002:86). Además, si lo pensamos bien, la diversidad de técnicas de comunicación, de búsqueda y de análisis de las dinámicas sociales desde el contacto directo con las personas en los diferentes niveles de relación humana (de lo individual a lo estructural) propias de la intervención social, tiene formas de articularse con la investigación en la búsqueda de significados y prácticas para generar valiosas reflexiones; cuáles son y cómo podemos hacer uso de algunas de tales herramientas se conforma como uno de los desafíos de los que daremos cuenta más adelante.

No podemos pasar por alto que ese mismo pragmatismo y la necesidad de respuesta rápida y medible ante situaciones de contingencia social que se le demanda a la profesión desde las instituciones en las cuales suele desarrollar la acción, la empuja a responder a los problemas sociales en tiempo real, exponiéndose al riesgo constante de caer en la instrumentalización y convertirse, en la práctica diaria, en un compendio de técnicas y recetas que pugnan por contestar de modo inmediatista a las necesidades particulares, en el mejor de los casos; sin embargo, como todo fenómeno complejo, la urgencia y la contingencia "es el marco de la dinámica del trabajo social profesional donde efectivamente los saberes se despliegan, se crean y recrean se incorporan y se resignifican, marcando el curso de la realidad que se aspira a interpretar" (Castañeda 2014:68).

Con todo lo anterior, debemos poner manos a la obra, como señala Toledo, para lograr "construir" pero "reestudiando el cuerpo de conocimientos existentes en las ciencias sociales" (2004:202). Se presenta en nuestro horizonte una importante serie de retos para conseguir tener herramientas, enfoques $y$ capacidad investigativa desde la particular visión del trabajo social, integrando una visión compleja. Planteamos, así, tres desafíos materializados en tres aspectos fundamentales de nuestra revisión - 
sujetos, objeto y metodología- que nos pueden ayudar a ir dando pasos hacia la construcción conjunta de una transdisciplina que investiga e interviene.

\section{Desafío I: Procesos inclusivos y colaborativos}

La dimensión ética es fundamental en una metodología y epistemología para el trabajo social. El primer desafío se presenta como la necesidad de hacer pesquisas y acciones guiadas por el enfoque de derechos, donde los trabajadores sociales somos garantes y promotores de participación y protagonismo social, resolviendo la investigación en un campo donde los actores sean convertidos en sujetos y agentes con visión sobre las situaciones y fenómenos que les acucian y con capacidad para construir conocimiento desde sus experiencias y saberes.

Para ello, la ética, que según autores como Castañeda ha podido sustituir a la epistemología: "Cuando en el discurso del trabajo social comienza a declinar la teoría, emergen como fundamento auxiliar los fundamentos éticos, filosóficos y humanistas que sirven como referentes éticos a la profesión" (2014:43), debe lograr expresarse en términos epistemológicos y ontológicos, constituyéndose en fundamento que dirija un proceso investigativo que nos permita comprender el mundo desde posiciones humanistas y comprometidas con las transformaciones sociales. De ahí que los estudios, de igual forma que se plantea en la práctica profesional, deben acoger los principios colaborativos y de participación como esenciales en la construcción de conocimiento desde la posición sujeto-sujeto como apuesta de relación entre los actores y que Vasilachis llama metaepistemología del sujeto conocido: "Se trata de considerar el resultado del proceso de conocimiento como una construcción cooperativa en la que los sujetos esencialmente iguales realizan aportes diferentes" (Vasilachis 2003:30); lo que desde otras disciplinas sociales se ha denominado "investigación reflexiva o dialéctica" (Canales 2006), "modelo del diálogo" (Clifford 2001) o "antropología de mutua transferencia" (Hernández 2003).

En diversas áreas teóricas y aplicadas, la confianza para el logro de esta inclusión ha sido puesta en las metodologías participativas. Estas se consideran como una forma posible de lidiar con el problema de la subjetividad y para la materialización práctica a través de la búsqueda de interpretaciones colectivas. Estas propuestas metodológicas se han conformado como IAP (Investigación Acción Participativa), que, en lo que a la integración de las personas se refiere, y en su modo más ortodoxo, supone la integración de los participantes en todas las fases del trabajo, desde principio a fin. No obstante, es necesario estar alerta: incluir técnicas de recogidas de informaciones interactivas y lúdicas podría llevarnos a asumir "ingenuamente de que el resultado es una autoría plural" (Ruano 2000:50). El uso de metodologías participativas no lleva irremediablemente a la igualdad en la construcción colectiva, puesto que, cuando se ejecutan, se ven cruzadas por relaciones de saber-poder todavía vigentes y arraigadas en las prácticas y los imaginarios.

Superar estas posiciones de saber-poder entre investigadores e investigados, más allá de las técnicas, requiere crear una relación diferente entre "nosotros" lejos de la dicotomía observador-observados y en base a comunicación activa, es decir, que se precisa una serie de principios y actitudes de interrelación que acompañen los encuentros, que favorezcan la actitud protagonista, la circulación de información y el diálogo. Ello precisa de un clima de seguridad que deje ser a los participantes, junto con apertura y flexibilidad para que los cambios ocurran. Al introducir grupos colaborativos, sociogramas, flujogramas, cartografía social y cualquier otra técnica promovida por los metodólogos de la IAP (especialmente los herederos de Fals Borda concretados en la escuela ibero-americana de Tomás Villasante), lo fundamental es sumar la idea del diálogo de James Clifford como proceso de intercambio, el cual 
considera la investigación como una negociación constructiva que involucra, por lo menos, a dos o más sujetos conscientes y políticamente significantes (Clifford 2001).

Cuando se integran estas claves y se realiza la construcción conjunta de los datos, hay consecuencias: se modifican sustancialmente las fases clásicas a las que estamos acostumbrados en los métodos de investigación social, donde trazamos objetivos e hipótesis y desempeñamos una serie de actividades para lograr alcanzarlos; puesto que el enfoque nos posibilita desviar la atención de lo ya producido y ajeno a nuestro dominio, que solo debemos medir o interpretar, para dirigirla a observar las relaciones sociales como procesos siempre en construcción, donde el mundo es asumido como espacios de transformación social colectiva. De este modo, si bien complica el trabajo, la construcción colaborativa ofrece la oportunidad de poner el énfasis en el proceso mismo de estudio, lo cual no significa que no pueda haber diseños previos, sino que estos se verán modificados, nutridos, a lo largo de su desarrollo.

Al asumir la igualdad de los actores, los sujetos son vistos como individuos sensibles y potentes en la narración de su propia historia, hábiles y legítimos. Esto supone que los participantes podrán añadir o cambiar los ejes de la exploración y las unidades de estudio, aportar al marco teórico desde los saberes y experiencias, incorporar preguntas investigativas, analizar los discursos e involucrase en todas la etapas que sea posible en una base de honestidad y respeto, donde la información es transparente, la inclusión es voluntaria, las decisiones se toman en común y los aportes son efectivamente tomados en cuenta.

\section{Desafío II: Incluir lo simbólico}

En aras de la inclusión de la dimensión simbólica a las investigaciones conectadas al trabajo social, deuda pendiente ya reclamada por varios autores (Matus 2002, Vélez 2003, Kisnerman 2006, Jiménez 2011), el segundo desafío lo constituye la incorporación de los elementos simbólico-culturales a nuestro objeto de estudio como dimensión irrenunciable para acercarnos a la complejidad de los espacios de interrelación social y los factores estructurales que los atraviesan. Este ámbito de producción y reproducción de significados es inherente a la realidad con la que se encuentra a diario el actuar disciplinar donde "las simbolizaciones se van creando a través de la mediación entre los sujetos en un espacio-tiempo que se comparte" (Carballeda 2002:97).

Pero evidenciar esta dimensión, es evidenciar también la necesidad de un proceso de análisis que permita conocer cuáles son sus vehículos y cómo estos generan y recrean órdenes y prácticas sociales, no solo en pos de la comprensión y la explicación, sino también de la intervención y las transformaciones sociales: "Las exigencias de cambio a través de las modificaciones de la práctica social, requiere una profundización en las representaciones sociales y en los significados que las personas otorgan a los actos y acciones que realizan cotidianamente" (Matus 2002:33). Para algunos autores esta dimensión es tan fundamental que "deberá constituir el núcleo claro o eje fundante de la reconfiguración disciplinar del trabajo social” (Vélez 2003:38).

En este resbaladizo terreno de lo intangible, debemos poner límites a qué vamos a comprender como dimensión simbólica y distinguir cuáles son los elementos que la componen. Solo concretando sus componentes, podremos poner en disposición algunas formas de ejecutar su estudio y análisis. Así pues, entendemos las representaciones sociales relacionadas con la idea de que las personas están inmersas en una red de significación subjetiva e imbuida por códigos, interpretaciones y usos propios y compartidos que se conforman en universos de representaciones simbólicas, donde "la representación es una parte esencial del proceso mediante el cual se produce el sentido y se intercambia entre los 
miembros de una cultura, esto implica el uso del lenguaje, de los signos y las imágenes que están por, o representan cosas" (Hall 1997:15).

Es a partir de esta idea, que las representaciones simbólicas se materializan en palabras e imágenes, desde donde la semiótica introdujo el estudio en profundidad del significado y el significante, del signo y su contenido cultural como forma de desentrañar los sentidos adscritos a cada imagen, signo o palabra. Sin embargo, va ser Foucault quien, en un intento de superación de la semiótica y del materialismo clásico, defina "el discurso" como una noción que conlleva, además, conflicto y construcción de la realidad con la transversalidad del poder: "El discurso define y produce los objetos de nuestro conocimiento, gobierna el modo como se puede hablar y razonar acerca de un tópico. También influencia cómo las ideas son puestas en práctica y usadas para regular la conducta de los otros mientras los sujetos son ubicados en relaciones de producción y de significación" (Foucault 1994:124). Por consiguiente, el discurso y su análisis se constituyen, en los estudios sociales, como un eje fundamental para lograr desentrañar los sentidos y significados en conexión con los contextos e intereses de determinados grupos. Van Dijk nos señala que hay tantos discursos como representaciones ideológicas compartidas: "Dependiendo de su posición, cada grupo seleccionará entre el repertorio de normas y valores sociales, propios de la cultura general; aquellos que realicen óptimamente sus fines e intereses, y se servirán de estos valores como los componentes que edifican sus ideologías de grupo" (1996:16).

En esta propuesta, vamos a asumir el discurso como elemento esencial de la dimensión simbólica, por cuanto nos permite una articulación micro y macro tan necesaria en nuestra área; no obstante, no vamos a quedarnos solamente en él, ya que se considera interesante sumar los ritos y los actos performativos. Los ritos y los rituales, apoyados por símbolos, son abordados como forma de performativizar las ideas. Las "expresiones performativas" ("performative utterances", que también se ha traducido por "expresiones realizativas"), es un concepto acuñado por John Austin desde la filosofía del lenguaje y, en esencia, se refiere a "lograr ciertos efectos por (el hecho) de decir algo" (Austin 1971:167). Debemos notar que "hecho" y "acto" se corresponden. El motivo por el cual se contemplan los actos simbólicos y la performatividad, es porque ellos nos conectan con lo ceremonial y el refuerzo de sentidos de realidad, de vínculos de identidad, de cohesión afectiva y de producciones de estatus, dominio y poder; aunque también pueden constituirse como creativos e imbuirse de nuevos sentidos y alianzas.

Discursos, símbolos, ritos y actos nos encaminan a la comprensión compleja de los espacios sociales de construcción simbólica-cultural con la mención del poder y su reproducción, pero también con los de creación, innovación y prácticas alternas. Sin embargo, incorporar estos elementos nos posiciona en una nueva encrucijada donde el reto investigativo lo constituye operativizar esta dimensión simbólica con fórmulas que resuelvan su concreción en los estudios.

Lo primero, en términos de resolución de lo que venimos hablando, es aplicar un enfoque multidimensional y sistémico, el cual obliga a ampliar nuestra capacidad de observación hacia niveles inmateriales de los fenómenos sociales con los que trabajamos, no de forma aislada, sino vinculadas con otras dimensiones de carácter práctico y estructural, estas últimas más familiares para nuestra profesión. Ello posibilita que pongamos atención en identificar y analizar las representaciones sociales compartidas socialmente que atraviesan nuestras instituciones, ver de qué manera se conjugan los discursos dominantes y qué imaginarios hay detrás de ellos; pero también aquellas representaciones que se producen y reproducen en sistemas más pequeños, como las familias o grupos particulares, e intentar 
profundizar en cómo todo ello se conforma en entramados complejos y recursivos de interacción y reconstrucción, en la que los trabajadores sociales juegan papeles fundamentales.

Como ejemplo de estos entramados complejos y recursivos, podemos tomar una doble unidad como identidad/necesidad, esto es, una necesidad o satisfactor determinado conectado a las adscripciones discursivas y las identificaciones que se hacen sobre los sujetos desde fuera como etiquetas, categorizaciones y estigmatizaciones. A través de esta relación, que parte de lo subjetivo e intersubjetivo, las personas gestionan sus identidades y otros elementos que intervienen en la construcción de los espacios donde vivir, en los contextos de significado y en las formas de convivencia compartidas o supuestamente compartidas. Así pues, esta relación necesidad/identidad podemos conectarla con lo macro y las estructuras, abriendo la oportunidad a la observación y el análisis de, por ejemplo, las acciones de participación social a los que los protagonistas se adhieren; las categorías en las ciudadanías; las leyes y políticas particulares a sectores designados étnicamente o focalizadamente, incluidas las discriminaciones positivas.

En segundo lugar, para el abordaje de nuestras unidades se requiere una configuración en varios niveles: micro, medio y macro, que nos va a proporcionar la delimitación de los sistemas que podrían estructurarse como unidades de observación (individuos, familias, grupos, comunidad, instituciones, etc.). Cada uno de estos niveles desplegará, a su vez, una serie de unidades de observación propias que va a depender de los alcances y las características del trabajo; pero, de un modo u otro, incluyen a los discursos, los ritos y las expresiones y actos performativos con sus lenguajes, imágenes y signos como vehículos sensibles de interpretación que puedan relacionarse con lo socialmente pensable, en el sentido de comprensión de su propia realidad, pero también de posibilidades de cambio y transformación de las situaciones-problemas que les acucian.

Una vez diseñadas las unidades de investigación, procede el registro y análisis del discurso, los actos performativos, ritos y los símbolos adscritos a ellas, los cuales serán recogidos con guías de observación en los que damos constancia tanto de los significados que otorgan los protagonistas, como de las posibles interpretaciones desde los observadores. Existen muchas alternativas para el análisis del discurso, algunas bien completas como las que propone la Escuela de Análisis del Discurso con modelos como el de Van Dijk; no obstante, para las guías de observación y análisis se propone recurrir a instrumentos y técnicas usadas en semiótica y antropología: guías de registro y análisis de ritos y actos y guías de observación de espacios, objetos e interacciones, todos ellos en base a marcadores simbólicos y/o lenguaje de las imágenes que los observadores podrán componer en pautas prácticas (1) o trascribiendo "descripciones densas" (Geertz) para ser sometidas al escudriñamiento simbólico.

La inclusión de esta dimensión simbólica en aras de una teoría de la intervención, nos mueve a una comprensión lo más cercana posible a las personas y su devenir social desde un aspecto tan inusitado como necesario en la (trans)disciplina. Esto permite captar significados y sentidos de las necesidades y problemáticas vinculadas a los que las vivencian, sus futuros posibles y los modelos y alternativas culturales y socio-políticos desde los cuales esas definiciones y demandas se hacen. En palabras de Vélez, su incorporación produciría "un cambio de perspectiva y darle cabida a la posibilidad de nombrar el mundo de lo intangible y lo posible" (2003:38), permitiendo una visión holística en la aprehensión de las dinámicas sociales. 


\section{Desafío III: Técnicas e instrumentos polivalentes}

Con la intención de complementar los dos anteriores desafíos materializados en sujeto y objeto, el tercer desafío se concreta en la acción de acercarnos a ellos con fines de comprensión y relación, conformando, de esta, una reflexión epistemológica que implica ética y técnica.

Así, esta última parte incursiona en el aprovechamiento de instrumentos de intervención propios del quehacer social con fines de estudio. Para ello requerimos, por un lado, de esa aceptación sin complejos de la práctica profesional como una transdisciplina donde vienen a converger enfoques y técnicas y, por otro, una observación y análisis cuidadoso de aquellos elementos que ya forman parte de nuestro corpus, para lograr convertirlas en instrumentos polivalentes o reversibles tanto para la práctica profesional como para la pesquisa investigativa.

De este modo, y como ya se ha señalado, la antropología es una excelente fuente de recursos de indagación social que son muy amigables con las lógicas de un acercamiento al conocimiento desde la práctica profesional, pudiéndose tomar métodos etnográficos y de carácter participante -observación directa y observación participante, entrevistas en profundidad, relatos de vida, cartas de parentesco, etc.-, así como instrumentos con los que podremos dar énfasis al análisis simbólico, tal como señalamos en el segundo desafío; pero, además, también pueden ser dispuestas en combinación con experiencias de la práctica profesional para lograr, como dice Carballeda "acceder a una explicación de la vida social que se ubica, en último término, en las experiencias vividas por los individuos" (2002:98).

La extensa experiencia en la intervención en situaciones de alta complejidad social que nos lleva a desplegar una multiplicidad de herramientas de comunicación y análisis de las dinámicas sociales y los contextos, es un rica fuente de recursos que puede permitir un espacio interesante y prolífico para rescatar significados, estructuras, praxis social y sentimientos adscritos para todos los participantes de un fenómeno social determinado; tal que la antropología nos posiciona de mejor manera ante lo simbólico, el trabajo social nos permite incidir en la dimensión práctica, en la acción cotidiana de los individuos y en sus sistemas inmediatos, así como crear canales de búsqueda dinámicos y novedosos que arrojan resultados de gran valor cualitativo.

Aunque las opciones que nos ofrece nuestra experiencia práctica son inmensas, a continuación, vamos a considerar cinco ejemplos para el uso investigativo de algunos de los instrumentos más emblemáticos del trabajo social.

Una de las actividades más identificadas con la profesión es la visita domiciliaria, definida por Tonon como: "Técnica de actuación que incluye la observación y la entrevista realizada en el domicilio de las personas y que facilita las interacciones en el lugar donde las personas tienen su vida cotidiana" (2005:57). Ya la autora hace alusión a los fines investigativos en su combinación de entrevista y observación, donde la especial ventaja la brinda el acercamiento a la intimidad y los territorios privados, tras, por supuesto, el acuerdo del uso del espacio y la voluntariedad a abrir sus domicilios por parte de los participantes. Esta técnica, tan controvertida, podría saldar su deuda social tras un uso como herramienta de intrusión y control de ciertos sectores de la población y conseguir instaurarse como una alternativa de encuentro entre sujetos, donde estos colaboran e intentan comprenderse desde su cotidianidad, tal como lo hace el etnógrafo cuando se sumerge en la comunidad. 
El análisis de casos o los estudios de casos en trabajo social, descrito por Berasategui como "lecturas de situaciones reales frente a las cuales cada uno ofrece su solución" (2009:52), proporcionan la oportunidad de comparar situaciones propias con las ajenas y analizarlas. Este recurso podría trascender a los profesionales y ser usado por los investigadores y los sujetos de investigación, conjuntamente, en el descubrimiento tanto de discursos y representaciones sociales, como de visiones compartidas de posible relación causa-efecto, entre otras opciones.

Desde la intervención sistémica podemos aprovechar diversos recursos, lo que se deja a merced de los dominios de cada uno y la responsabilidad sobre los alcances de la técnica. Desde aquí, proponemos la entrevista sistémica, también llamada "en estrella" o "circular", como una forma interrelacionada de conocer sentimientos y significados otorgados a sucesos y actos de los miembros de un sistema. La entrevista sistémica nos permite una entrevista compleja a familias y grupos en la que los componentes dan y escuchan repuestas en las que los otros quedan incluidos como parte del relato. Este tipo de técnica permite, además, triangular las observaciones de varios sujetos sobre un mismo hecho o situación en una misma actividad.

El rol playing o juego de roles es otro de los recursos apropiados por la profesión que ha ido adquiriendo un gran espectro de usos en la intervención y que tiene bastantes potencialidades a la hora de hacer un estudio. El rol playing comunica en una simulación a dos o más personas representando una situación y asumiendo roles. El cuerpo y/o la palabra se constituyen como los vehículos de expresión de situaciones que, en ocasiones, son difíciles de narrar y, además, por su particularidad, se revela como una forma inclusiva de personas iletradas y niños que pueden "actuar" su visión sobre una situación dada, en lugar de intentar explicarla.

Por último, remitimos como herramientas de mapeo de relaciones el genograma y el ecomapa, considerándolos como un acercamiento menos cualitativo pero útil por su disposición de información gráfica y relacional. No obstante, si los combinamos con la carta de parentesco utilizada en etnografía adquieren mucha mayor riqueza. Esta hibridación de la carta de parentesco, el genograma y sociograma, permite, a partir de un sujeto, identificar patrones socio-económicos y profesionales, matri y patrilocalidades, patri y matrilinealidades y algunos referentes interesantes de movilidad territorial y conexiones transnacionales, como la historia migratoria de una familia.

Todos estos ejemplos, son una muestra de las posibilidades que las actividades utilizadas en el diagnóstico y la intervención pueden tener una vez articulados a los objetivos investigativos. Considerando que pueden irse modificando a merced de la osadía y la creatividad de los participantes del estudio, en general, todas ellas, más allá de la recogida de información, se presentan como fructíferas en un planteamiento inclusivo y colaborativo de la investigación, facilitando una apertura a la discusión y la construcción conjunta. Definitivamente, desde nuestra propuesta, todas las alternativas expresadas son impensables sin una comunión con los sujetos en el proceso y la garantía de derechos explicitada en el primer desafío: los participantes deben no solo conocer y apoyar el uso de una actividad concreta, sino también deben corresponsabilizarse de ella.

Por último, señalar que comprendiendo y asumiendo la transdisciplinariedad del trabajo social podemos seguir alimentando nuestra reserva de instrumentos y técnicas de investigación, cada uno acogiendo los riesgos que vea posible asumir; pero, también, se plantea como una apuesta bidireccional, en la que podemos evidenciar al resto de las disciplinas que disponemos de una rica gama de recursos utilizados para el diagnóstico y la intervención que pueden ser adaptados como útiles investigativos, como forma 
de encontrarnos de manera distinta y explorar nuevos modos de relacionarnos con la aprehensión de la realidad y los sujetos que la viven y construyen.

\section{Conclusiones}

En nuestra profesión, nos encontramos con los retos investigativos y epistemológicos bajo la exigencia de "superar el binarismo conocer-hacer" (Matus 2002:14), que conforme una "acción social dialogante e interactiva" (Vélez 2003:23). Ello, debemos asumirlo como desafíos a los que responder, es decir, no limitarnos a adscribirnos, sin más, a los paradigmas vigentes en ciencias sociales, sino atreviéndonos a aportar desde nuestra experiencia.

La exploración investigativa ha llevado al trabajo social, en algunas academias chilenas, no solo a emular la investigación sociológica, sino que en pro del reconocimiento de "cientificidad" se produce una inclinación hacia los estudios cuantitativos de corte positivista obviando la crisis del modelo. No se tiene que renunciar a lo medible, que podría ser interesante dependiendo de la naturaleza de los estudios que se emprendan; no obstante, las características del trabajo social lo maridan mucho más armoniosamente con lo cualitativo, con metodologías flexibles e inclusivas que faciliten rescatar la posición privilegiada de relación con la gente y las dinámicas cotidianas.

Desde esta propuesta, queremos aportar a la vigilancia epistemológica desde términos éticos y metodológicos, como también ontogénicos, esto es, no solo constituyéndonos desde lo que ya somos, sino desde lo que podemos ir siendo, en gerundio, navegando hacia posibilidades por abrir y descubrir, novedosas e interesantes, transdisciplinadas, formas de reinventarnos.

Las proposiciones expuestas también son compatibles con estudios en profundidad enmarcados en instituciones de intervención social y prácticas profesionales, lo que es un riesgo y una oportunidad a la vez. La ventaja es la excelente posición de observación y relación con los sujetos, con confianzas superadas y seguridad para conocer y transitar los posibles caminos investigativos; pero un riesgo por cuanto el trabajador social puede ser una parte del engranaje institucional, tan inserto que naturalice los valores e imaginarios que rigen las políticas públicas y la acción social, justificando la extensión y las características de los elementos más estables, asumidos como propios. También puede mostrarse resistente a analizar los fundamentos de su trabajo, donde las relaciones con los sujetos pueden estar minadas desde vínculos clientelistas y/o paternalistas. Por ello, parte de la clave consiste en que el trabajador social investigador sea asumido como sujeto y objeto de todo el proceso, en el cual se debe ubicar como agente en dichas prácticas revisando sus categorías conceptuales, los términos que usa para los análisis, las caracterizaciones humanas que utiliza para designar y, por supuesto, los significados tras las iniciativas de acción social.

Las reflexiones de lo que podemos y debemos ser nos han llevado a querer configurarnos como algo más allá que un corpus tecnológico. Desde aquí, se comprende que requerimos aprovechar las ventajas de estar en primera línea social, en contacto privilegiado con las personas, y conseguir llegar a ser la articuladora de reflexión/acción por excelencia, fuente legítima de una teoría de la intervención y ser reconocida así por el resto de las ciencias sociales. Una teoría de la intervención se debe plantear como dinámica, viva, siempre alerta y polisémica, basada en un entendimiento de las necesidades, desigualdades y las acciones sociales desde la vivencia cotidiana, con la inclusión de sus protagonistas, donde la información está al servicio de las personas, los colectivos y los pueblos, para la garantía de los derechos políticos, civiles, sociales, culturales y ecológicos. 
Una teoría de la intervención no puede, ni debe, constituirse como un recetario, sino que su fin es aportar a la visibilización de lo omitido, lo subalterno, lo alter-nativo, así como deconstruir formas de análisis de lo social anquilosadas en el trabajo social y en las ciencias sociales de acción: "Acercarnos a propuestas que ofrezcan alternativas para la construcción de nuevos idearios sobre los que abrir campos de reflexión y análisis de las formulaciones e intervenciones en el campo de la acción social" (Ortega 2015:323). Por ello, es que debemos trazar metas y proveernos para asumir desafíos que favorezcan una complementariedad interesante entre la investigación y la intervención, procurando caminos por los que indagar formas de conseguir conocimientos que alumbren cómo dar respuesta a los problemas sociales, y que imagine futuros que aporten a la comprensión de los fenómenos más allá de la sistematización, sin excluirla.

\section{Nota}

(1) Los instrumentos fueron utilizados por los estudiantes en su aprendizaje en la asignatura "Análisis metodológicos de Etnias" de la carrera de Trabajo Social de la UCSC. La autora pone a disposición del que lo requiera guías de observación y de registro en base a marcadores simbólicos utilizados en la investigación de la que surgen las reflexiones de este artículo, escribiendo al correo mbortega@ucsc.cl

\section{Bibliografía}

Aquín, N. 2006. Reconstruyendo lo social. Prácticas y experiencias de investigación desde el trabajo social. Buenos Aires: Editorial Espacio.

Austin, J. L. 1971. Como hacer las cosas con palabras. Palabras y acciones. Barcelona: Paidós Studio.

Berasategui, A. 2009. Técnicas grupales: dinámicas en pequeños y grandes grupos. En: C. Guinot. Métodos, técnicas y documentos utilizados en trabajo social. Bilbao: Deusto Publicaciones, pp. 43-58.

Carballeda, A. 2002. La intervención en lo social. Exclusión e integración en los nuevos escenarios sociales. México: Paidós.

Castañeda, P. 2014. Propuestas metodológicas para el trabajo social en intervención social y sistematización. Santiago: Universidad de Valparaíso.

Camelo, A. y Cifuentes, R.M. 2006. Aportes para la fundamentación de la intervención profesional en trabajo social. Revista Tendencias \& Retos 11: 169-187.

http://revistas.lasalle.edu.co/index.php/te/article/view/1596/1472

Canales, M. 2006. Metodologías de investigación social. Introducción a los oficios. Santiago: LOM

Cifuentes, R.M. 2009. Consolidación del trabajo social en las ciencias sociales: desafío y horizonte en la formación profesional en Colombia. Eleuthera 3: 40-71.

Clifford, J. 2001. Dilemas de la cultura. Barcelona: Gedisa. 
Escobar, A. 1999. Antropología y desarrollo. Revista Maguaré 14: 42-73.

http://www.revistas.unal.edu.co/index.php/maguare/article/view/11135/11803

Foucault, M. 1994. Power: the essential works of Michel Foucault 1954-1984. London: Penguin.

Hall, S. 1997. Representation. London: Sage.

Hernández, I. 2003. Autonomía o ciudadanía incompleta: el pueblo mapuche en Chile y Argentina.

Santiago: Ed. Pehuén.

Jiménez, C. 2011. ¿Empobrecimiento o desclasamiento? La dimensión simbólica de la desigualdad social. Trabajo y Sociedad 17: 49-65.

Kisnerman, N. 2006. Pensar el trabajo social. Una introducción desde el construccionismo. Buenos Aires: Edward.

Matus, T. 2002. Propuestas contemporáneas en trabajo social: hacia una intervención polifónica. Buenos Aires: Espacio Editorial.

Nicolescu, B. 1996. La transdisciplina. México: Edición 7 saberes.

Ortega, M.B. 2015. El cuestionamiento del poder como dominio: repensar los análisis de poder en la acción social. Tabula Rasa 22: 323-334. revistatabularasa.org/numero-22/16ortega.pdf

Ortega, M.B. 2013. Sistemas, tridimensionalidad y deconstrucción: aplicando complejidad a la investigación social antropológica. Revista Mad (28): 61-70. doi: 10.5354/0718-0527.2013.26948

Quiroz, M.H. 2010. El aporte de Edgard Morín. Trabajo social y pensamiento complejo. En: S. Iturrieta y D. Sánchez. Perspectivas de trabajo social: Reflexiones acerca de la disciplina. Santiago: Ediciones Universidad Católica Silva Henríquez, pp. 157-173.

Ruano, L. 2000. De la construcción de los otros por nosotros a la construcción del nos-otros. Educar 12: 46-58.

Saavedra, J. 2015. Cuatro argumentos sobre el concepto de intervención social. Cinta moebio 53: 135146. doi: $10.4067 /$ S0717-554X2015000200003

Sousa Santos, B. 2003. Um discurso sobre as ciências. Porto: Ediçôes Afrontamento.

Toledo, U. 2004. ¿Una epistemología del trabajo social? Cinta moebio 21: 200-214.

moebio.uchile.cl/21/toledo.htm

Tonon, G. 2005. Las técnicas de actuación profesional del trabajo social. Buenos Aires: Espacio.

Van Dijk, T. 1996. Análisis del discurso ideológico. Versión 6: 15-43.

Vasilachis, I. 2003. Pobres, pobreza, identidad y representaciones sociales. Barcelona: Gedisa.

Vélez, O. 2003. Reconfigurando en trabajo social. Perspectivas y tendencias contemporáneas. Buenos Aires: Espacio ed.

Recibido el 25 Mar 2015

Aceptado el 8 Jun 2015 